Meta

Journal des traducteurs

Translators' Journal

\title{
La traduction du japonais
}

\section{Norman Bernier}

Volume 11, numéro 3, septembre 1966

URI : https://id.erudit.org/iderudit/010473ar

DOI : https://doi.org/10.7202/010473ar

Aller au sommaire du numéro

Éditeur(s)

Les Presses de l'Université de Montréal

ISSN

0026-0452 (imprimé)

1492-1421 (numérique)

Découvrir la revue

Citer cet article

Bernier, N. (1966). La traduction du japonais. Meta, 11(3), 79-84.

https://doi.org/10.7202/010473ar

Ce document est protégé par la loi sur le droit d'auteur. L’utilisation des services d'Érudit (y compris la reproduction) est assujettie à sa politique d'utilisation que vous pouvez consulter en ligne.

https://apropos.erudit.org/fr/usagers/politique-dutilisation/
Cet article est diffusé et préservé par Érudit.

Érudit est un consortium interuniversitaire sans but lucratif composé de l'Université de Montréal, l'Université Laval et l'Université du Québec à Montréal. Il a pour mission la promotion et la valorisation de la recherche. https://www.erudit.org/fr/ 


\section{LA TRADUCTION DU JAPONAIS}

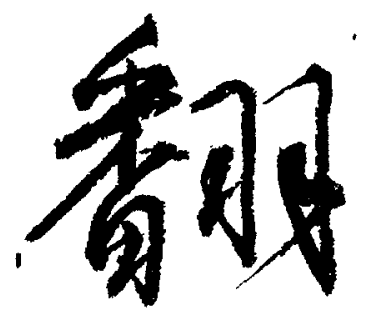

Honyalku se rend en français par "traduire». Il se compose de deux caractères principaux: hon et yaku. Hon est à son tour formé de deux caractères: tsugaeru (unir, accoupler) et hane (ailes d'oiseau). Quant yaku, il est formé de kotoba (mot) et de shaku (mesurer).

«Par des chemins divers, les hommes peuvent gravir la montagne,

Chaque sentier découvre des voies différentes, sublimes;

Mais quand ils atteignent au fier sommet,

C'est la même lune souriante qui charme leurs regards. »

(Dr. KaTO)

De ces sentiers divers, de ces voies différentes surgissent maintes difficultés pour un traducteur de textes japonais. En règle générale, l'éclairage particulier que la langue japonaise projette, par exemple, sur l'honneur, la politesse, l'amour, la famille, n'est guère familier aux Occidentaux.

Ainsi, l'honneur, pour un Japonais, c'est le bien suprême sur terre. Le Japonais n'hésitera même pas à donner sa vie pour sauver sa réputation. L'honneur n'est ni plus ni moins que la conscience japonaise.

Et la politesse? Loin d'être un épiphénomène, c'est au Japon un art dont le but est de cultiver l'esprit, d'en montrer la supériorité sur la chair. Il est certes plus facile de sourire à la vue de nombreuses courbettes que de découvrir les racines profondes de la politesse japonaise.

Un dicton populaire comme «Joie triste de la lune, de la neige et des fleurs » évoque immédiatement, du moins pour un Japonais, l'idée suivante: la contemplation de la nature console des malheurs.

La connaissance des nombreux dictons japonais qui forment l'épitomé de la morale sociale permet au traducteur de se rendre compte du rôle important que l'esthétique joue dans la vie du Japonais. Elle lui révèle l'attirance instinctive du Japonais vers la beauté, son aptitude à apprécier rapidement formes et couleurs, son sentiment pour l'élégance simple, son habitude à trouver le bonheur dans des choses communes et - facteur de toute première importance pour un traducteur - sa démarche intuitive et sensible ainsi que sa méfiance de la logicue et de l'analyse. 
Que cette connaissance provienne de l'expérience ou d'études systématiques importe peu. L'essentiel est qu'elle soit présente à l'esprit quand le traducteur aborde son texte.

Autrement les faussetés et les sottises règnent en maîtresses même dans la traduction d'un seul idéogramme. Ainsi, le terme kami, attaché à la personne de l'empereur, en a vu de toutes les couleurs. Que des «touristes » rendent ce caractère par dieu ou Dieu ne surprend guère; d'ailleurs, certains dictionnaires optent pour cette solution de facilité. Mais que fait-on des conséquences? Dire que l'empereur est Dieu, alors qu'il n'est qu'un kami, c'est fausser la réalité. Le Japonais n'a jamais pensé que l'empereur était un dieu, créateur tout-puissant et éternel.

Kami veut dire «au-dessus, sur » et veut donner une idée de ce qui est supérieur, élevé comme la cime d'une montagne, le haut d'une vallée, les cheveux de la tête. Il indique ceux qui gouvernent les êtres surnaturels, l'empereur, les héros, les choses invisibles, les forces secrètes ... Le terme kami s'applique tout aussi bien à des êtres humains supérieurs qu'à des oiseaux, des plantes, des arbres, des montagnes et autres choses dignes d'être révérées pour les pouvoirs exceptionnels qu'elles possèdent.

En ce sens, kami serait plutôt un reflet de la divinité, une parcelle du divin, une goutte plus concentrée de ce divin que l'on retrouve partout dans les choses; il y a de même une différence assez grande entre un reflet de la divinité et la divinité elle-même, tout comme les catholiques ne qualifient pas le pape de «Dieu » du fait qu'il est revêtu de l'infaillibilité ou qu'il est le Vicaire du Christ.

Le traducteur se rend compte bien vite que notre écriture correspond mal au langage des idéogrammes japonais.

La structure grammaticale du japonais cache également plus d'un piège pour un esprit français. Prenons l'exemple d'une phrase écrite en japonais romanisé ${ }^{1}$ :

\section{Senshuu no/eiga wa/puroguramu ga/suki dewa nakatta shi/hima mo/nakatta no de/ikimasen deshita.}

Traduction littérale: Semaine dernière de/film/programme/aimer pas avoir eu/et/temps aussi/pas avoir eu parce que/pas aller avoir été.

Il saute aux yeux que les différences entre le japonais et le français sont nombreuses.

D'abord, l'ordre des mots dans la phrase correspond peu à notre façon de voir. Le verbe de la proposition principale est toujours placé à la fin de la phrase, le déterminatif toujours avant le déterminé. Cette façon de procéder à l'inverse du français est tellement courante qu'il vaut mieux en prendre son parti tout de suite. La première page d'un livre japonais ne correspond-elle pas à la dernière d'un livre français? Le Japonais semble pour nous commencer à lire par la fin. De plus, le texte se lit de droite à gauche et de haut en bas.

1. Grâce à cette romanisation, il est possible d'exprimer le japonais en caractères latins et par conséquent de le citer dans un texte occidental. Normalement, le japonais s'écrit au moyen de caractères chinois (idéogrammes) et de syllabaires japonais (katagana et hiragana). 
Si nous relisons la phrase précitée en commençant par la fin, nous aurons: [Je] ne pas être allé [voir le film] parce que [je] ne pas avoir eu temps et [que je] ne pas avoir aimé programme de film de semaine dernière.

En rétablissant les liaisons syntaxiques normales, on obtiendra la traduction suivante: «Je ne suis pas allé au cinéma la semaine dernière parce que je n'en ai pas eu le temps. D'ailleurs, le programme ne me plaisait pas.»

Un examen plus détaillé de l'exemple susmentionné fait ressortir les différences suivantes:

L'article - Il n'existe pas en japonais. Seul le contexte indique si le nom est défini ou non.

Le nombre - Le nom japonais ne l'exprime pas. Eiga (film) est invariable. Ce n'est que le contexte qui peut nous fournir une indication sur le nombre. Dans quelques cas, on peut exprimer le pluriel en ayant recours à un suffixe (watakushi $=$ moi, wakushitachi $=$ nous $)$ ou en répétant le nom $(\mathbf{k u n i}=$ pays, kuniguni $=$ les pays $)$.

Le genre - En principe, la morphologie du japonais n'exprime pas cette catégorie. Eiga $(f i l m)$ n'est donc ni masculin ni féminin. Pour exprimer les différences de sexe, le japonais utilise des noms différents comme en français (chichi $=$ père, haha $=$ mère $)$ ou des noms asexués.

Les particules - Contrairement au français qui utilise abondamment des prépositions, le japonais se sert de postpositions qui indiquent la fonction grammaticale du substantif qui les précède: no, le génitif; ga, le cas sujet; wa, le cas absolu; wo, l'accusatif.

Le verbe - Ikimasen deshita est le verbe de la proposition principale. Il ne marque ni le nombre ni la personne. Le contexte seul fournit ces précisions. La terminaison masen indique que le verbe iku (infinitif) est à la forme négative. Le verbe a en effet une terminaison différente selon qu'il exprime une négation ou une affirmation. Deshita est le passé de l'auxiliaire desu; son usage permet de rendre nettement lidée de passé. En revanche, le présent et le futur se confondent.

L'emploi de termes grammaticaux français pour désigner des réalités japonaises ne sert qu'à des fins de comparaison. Parler de conditionnel ou d'impératif à propos du japonais, c'est évoquer des idées bien différentes du français. Le conditionnel japonais fonctionne en fait à l'inverse du conditionnel français. Il spécifie le verbe de la proposition conditionnante, non celui de la proposition conditionnée:

Kono kusuri nomeba kimochi yoku naru deshoo.

Traduction littéralè: Ce médicament prendriez santé bien devenir peut-être.

Tournure japonaise: Si vous prendriez... vous devenez...

Traduction: «Si vous prenez ce médicament, vous vous sentirez mieux». 
Le conditionnel japonais peut aussi désigner le temps et se rend par « quand».

Kono yoji ga sumeba kaette kite kudasai.

Cette affaire / finir quand / retourner venir veuillez.

« Revenez quand vous aurez fini cette affaire».

« Revenez » ne se rend pas en japonais par un impératif, à moins de faire fi de la politesse. Pour exprimer le commandement, on emploie presque toujours un auxiliaire de commandement: kudasai, équivalent du français «veuillez ».

La politesse est tellement nuancée qu'elle pousse le Japonais à rendre, par exemple, le verbe «donner » de nombreuses façons, selon qu'il parle de lui-même (forme humble) ou qu'il s'adresse à un interlocuteur (forme neutre ou forme honorifique).

\begin{tabular}{|lll|}
\hline forme humble & forme neutre & forme honorifique \\
ageru & yaru & kudasaru \\
sasageru & ataeru & hodokosaru \\
kisosuru & jureru & gokashi ni naru \\
shinteisuru & sazukeru & keiyosaru \\
& & osazuke ni naru \\
& osazuke tamoo \\
& osazuke nasaru \\
& osazuke asobasu \\
& etc. \\
\hline
\end{tabular}

On pourrait expliciter par un diagramme les rapports sociaux qui se traduisent par l'emploi de formes verbales différentes:

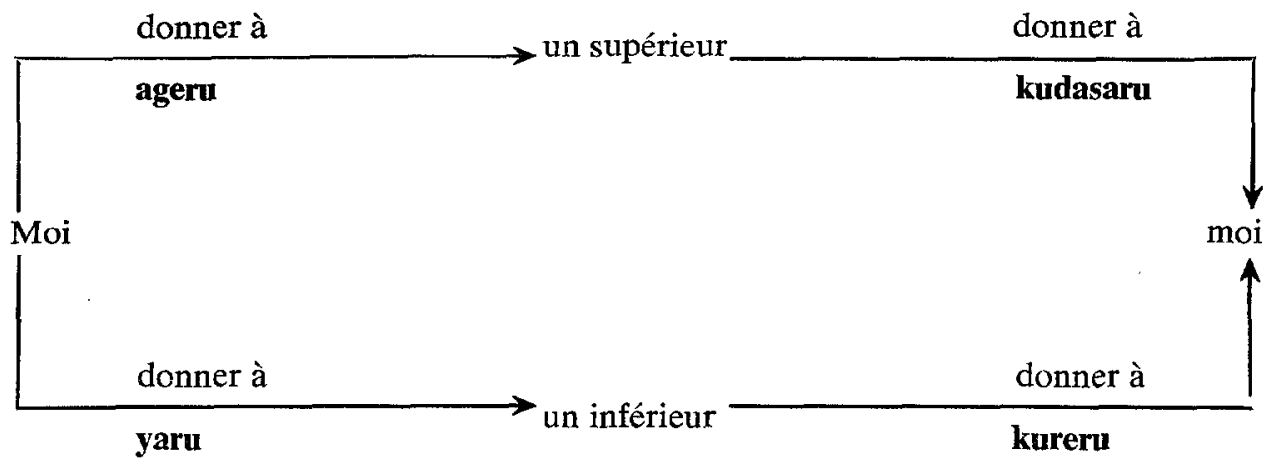

Ainsi le verbe ageru signifie beaucoup plus que l'idée de «donner », il précise que c'est moi qui donne à un supérieur (patron, professeur, ministre). En revanche, si c'est un supérieur qui s'adresse à moi, il devra utiliser le verbe kudasaru pour exprimer la même idée de «donner». 
En plus de refléter des degrés de politesse ou d'humilité, ces verbes servent à distinguer les actions et les choses de la première, de la deuxième ou de la troisième personne. Par la même occasion, ils révèlent le rang social des locuteurs; pour bien les traduire, il faudra se référer au contexte.

On peut se demander justement si ce besoin de référence continuelle au contexte ne trahit pas la confusion et l'imprécision de la langue. La question est d'autant plus importante qu'elle nous place d'emblée sur le plan de la stylistique japonaise. Comme le dit le célèbre écrivain Junichiro Tanizaki dans son traité sur le style littéraire, le vague est plus qu'une qualité de l'écrivain: «L'écrivain japonais doit faire du vague une vertu. À la clarté, il doit préférer le clair-obscur; à la concision, la périphrase. 》

Cette conception de Tanizaki explique davantage la préférence du japonais pour les périphrases, les formules indirectes et atténuées, les expressions négatives, les phrases interrogatives-négatives.

Que le japonais ait une prédilection pour les périphrases n'est pas difficile à démontrer. Une phrase aussi simple que « ce n'est pas froid » se rend en japonais par:

Variantes Explications

1. Samuku nai

2. Samuku arimasen

3. Samuku nai no desu

4. Samuku nai n desu

5. Samui no de wa nai

6. Samui n ja nai

7. Samui no de wa arimasen

8. Samui no de wa nai no desu

9. Samuku wa gozaimasen langue parlée, forme neutre utilisée par les hommes et les femmes.

langue parlée, forme plus polie que la précédente, utilisée par les hommes et les femmes.

langue parlée, référence à la personne qui parle.

langue parlée, forme plus familière que la précédente, utilisée par les femmes.

langue littéraire.

forme plus familière que la précédente, utilisée par les femmes.

référence à une tierce personne, forme polie.

une tierce personne donne l'impression d'avoir froid, mais elle n'a pas froid. réponse polie, utilisée par les femmes.

«Un enfant intelligent » se rend par wakari no hayai kodomo wa. La traduction littérale donnerait ceci: Un enfant dont l'intelligence est prompte.

«Un homme malchanceux » par un no warui hito wa, à savoir: Un homme dont la chance est mauvaise. 
Il ne faudrait pas croire, d'après nos traductions, que le japonais est friand de relatives. En fait, le pronom relatif n'existe pas en japonais. On traduit comme s'il était toujours sous-entendu. Monsieur Tanizaki ne nous a-t-il pas révélé que le vague est une vertu?

Un dernier exemple pour illustrer, cette fois, la préférence du japonais pour les formes négatives:

《Vous devez écrire avec un stylo.»

Pen de kakanakute wa ikemasen.

Avec un stylo / si vous n'écrivez pas / ça ne marche pas.

Ces différences peuvent sembler éloigner les hommes les uns des autres. Elles ne sont pourtant que les voies divergentes permettant d'atteindre au fier sommet où

«C'est la même lune souriante qui charme leurs regards.»

NORMAND BERNIER

Montréal 